\title{
Trabajo colaborativo en línea como estrategia de aprendizaje en entornos virtuales: una investigación con estudiantes universitarios de Educación Infantil y Educación Primaria
}

ALMUDENA COTÁN FERNÁNDEZ*

Universidad de Cádiz - Espańa

IRENE GARCÍA-LÁZARO*

Universidad Internacional Isabel I - Espańa

JOSÉ GALLARDO-LÓPEZ

Universidad Pablo de Olavide - Espańa

Recibido el 19-03-20; primera evaluación el 04-01-21; segunda evaluación el 24-01-21; aceptado el 28-02-21

\footnotetext{
* Doctora en Educación por la Universidad de Sevilla. Actualmente es profesora en la Facultad de Ciencias de la Educación de la Universidad de Cádiz. Ha desempeñado otros puestos docentes en la Universidad de Sevilla, Fundación CEU San Pablo Andalucía y Universidad Isabel I. Sus intereses de investigación se centran en Educación Superior, estudiantes con discapacidades, pedagogía inclusiva, TIC, formación de los profesores, innovación, Diseño Universal de Aprendizaje e investigación cualitativa. Correo electrónico: almudena.cotan@uca.es. https://orcid.org/0000-0003-0362-4348

** Doctora en Pedagogía por la Universidad de Sevilla. Se desempeña en la Universidad Internacional Isabel I como profesora en la Facultad de Ciencias de la Salud y Facultad de Humanidades y Ciencias Sociales. Ha desempeñado otros puestos docentes en la Universidad de Sevilla. Sus inquietudes investigadoras se centran en las nuevas tecnologías aplicadas a la educación, orientación educativa, convivencia en la escuela y educación emocional. Correo electrónico: igarlaz@gmail.com. https:// orcid.org/0000-0001-6000-8424

${ }^{* * *}$ Doctor en Pedagogía por la Universidad de Sevilla. Profesor de la Universidad Pablo de Olavide de Sevilla, adscrito al Departamento de Educación y Psicología Social. Profesor de la Universidad Internacional Isabel I en la Facultad de Humanidades y Ciencias Sociales. Entre sus principales líneas de investigación se encuentran: nuevas tecnologías aplicadas a la educación, inteligencia emocional, pedagogía social, coeducación y atención a la diversidad funcional. Correo electrónico: jagallop@upo.es. https://orcid.org/0000-0003-3243-1676
} 


\section{RESUMEN}

Este artículo se centra en los resultados de un estudio realizado en una Universidad privada de España en la que se analizaron las principales potencialidades, beneficios e inconvenientes del trabajo colaborativo en entornos en línea. Concretamente, se estudió el trabajo colaborativo como estrategia de aprendizaje y cómo influía en el proceso de adquisición de conocimientos de los estudiantes. Partiendo del método cualitativo, se utilizó la entrevista estructurada como instrumento de recogida de información. Los resultados de este trabajo analizan cómo el trabajo colaborativo influye en el aprendizaje de los estudiantes y cuáles eran las modalidades de organización más significativas, entre otros aspectos. Entre las principales conclusiones obtenidas, cabe resaltar cómo la implicación personal y la responsabilidad individual son aspectos esenciales para generar un buen clima de cohesión grupal.

Palabras clave: educación virtual, educación Superior, estrategias de aprendizaje, aprendizaje colaborativo, trabajo en equipo.

\section{Online collaborative work as a learning strategy in virtual environments: an investigation with university students of Infant and Primary Education}

\section{Abstract}

This article presents the results of study carried out at a private university in Spain which the main potentialities, benefits and disadvantages of collaborative work at online environments were analyzed. We studied collaborative work as a learning strategy and how it influenced the process of knowledge acquisition of students. Starting from the qualitative method, the structured interview was used as an instrument to collect information. The results of this work analyze how collaborative work influences student learning and what were the most significant organizational modalities, among other aspects. The main conclusions obtained, it is worth highlighting how personal involvement and individual responsibility are essential aspects to generate a good climate of group cohesion.

Keywords: Virtual education, higher education, learning strategies, collaborative learning, teamwork.

\section{Trabalho colaborativo on-line como estratégia de aprendizagem em ambientes virtuais: uma investigaçáo com estudantes universitários da Educaçáo Infantil e Primária}

\section{Resumo}

Este artigo enfoca os resultados de um estudo realizado em uma universidade privada na Espanha, no qual foram analisadas as principais potencialidades, benefícios e desvantagens do trabalho colaborativo em ambientes on-line. Especificamente, o trabalho colaborativo foi estudado como estratégia de aprendizagem e como ele influenciou o processo de aquisição de conhecimento dos estudantes. 
Com base no método qualitativo, a entrevista estruturada foi usada como um instrumento para coletar informaçóes. Os resultados deste trabalho analisam como o trabalho colaborativo influencia o aprendizado dos estudantes e quais foram as modalidades organizacionais mais significativas, entre outros aspectos. Entre as principais conclusóes obtidas, vale destacar como o envolvimento pessoal e a responsabilidade individual são aspectos essenciais para gerar um bom clima de coesão grupal.

Palavras-chave: Educação Virtual, Educação Superior, estratégias de aprendizagem, aprendizagem colaborativa, trabalho em equipe.

\section{INTRODUCCIÓN}

En las últimas décadas se ha apreciado un auge de las instituciones de Educación Superior en línea. El modelo educativo establecido por el Sistema Universitario Español al Espacio Europeo de Educación Superior (EEES), ha evidenciado cambios sustanciales en las universidades (Algaba, 2015), especialmente en su modalidad de enseńanza: presencial, semipresencial o en línea. Cabe destacar las cifras obtenidas en el último informe elaborado por Hernández y Pérez (2019), donde indican que, durante el curso 2017/2018, la matrícula en estudios no presenciales aumentó un $85 \%$ en las universidades públicas y un $90 \%$ en las universidades privadas. Este crecimiento por parte de las universidades para ofrecer formación en línea está relacionado con la flexibilidad que ofrece esta modalidad de estudios, pudiéndose combinar la vida personal con la profesional. A su vez, la inclusión de una amplia cobertura de estudiantes, la extensa oferta formativa que ofrece, el fácil y rápido acceso al contenido y materiales didácticos por parte del alumnado y su adaptación a las necesidades personales, familiares y laborales, son aspectos que inciden en este crecimiento (Cotán et al., 2020).

Esta realidad presenta un cambio en la forma tradicional del aprendizaje por parte del estudiante. Se ha pasado de las lecciones magistrales sin intervención por parte del alumno a un modelo centrado en el aprendizaje activo e interactivo que le dota de mayor protagonismo y responsabilidad (Mostrom y Blumberg, 2012). Unido a ello, diversos estudios (Santizo, 2016; Chang y Kang, 2016; Gallardo-López y López-Noguero, 2020) han dejado patente la eficacia de las metodologías interactivas, tan válidas como las tradicionales. Por ello, en este trabajo se analiza algunas de las estrategias de aprendizaje que, desde los entornos en línea, se desarrollan para el logro del aprendizaje del estudiante. De manera específica, se centrará en el desarrollo del trabajo colaborativo y sus implicaciones. 


\section{Marco teórico}

Los espacios virtuales de formación ofrecen diversas posibilidades para el desarrollo de actividades y de los aprendizajes. El trabajo colaborativo, la interacción y participación por parte del estudiante y las acciones tutoriales, son estrategias y actuaciones que permiten adquirir las competencias y los objetivos establecidos en sus titulaciones.

En este contexto, la colaboración entre estudiantes es considerada un elemento esencial del aprendizaje en línea (Cotán et al., 2020; Molina, 2013). Es tal su importancia que, desde el proyecto Tuning Educational Structures in Europe (González y Wagenaar, 2003) se establece el trabajo en equipo como una de sus principales competencias. En este entorno, el aprendizaje producido a través de esta estrategia es significativo al encontrarse bajo una perspectiva «situativa» (Marín et al., 2018) donde el alumnado genera oportunidades de aprendizajes permanentes, desarrollando discusiones profundas (Korhonen et al., 2019; Castellanos y Niño, 2020) a través del contraste de perspectivas y opiniones, así como de su propia implicación y compromiso (Yadegaridehkordi et al., 2019; Zhou et al., 2019). Esto permitirá conectar el aprendizaje del alumnado con situaciones reales.

Este tipo de estrategia didáctica y formativa estimula a que el alumnado desarrolle habilidades comunicativas, relaciones simétricas y recíprocas y genere espacios de interacción y aprendizaje (Erkens y Bodemer, 2019; Kim y Ketenci, 2019; Chang y Kang, 2016). De hecho, cuando el alumnado comparte sus opiniones y objetivos, hace que el grupo pueda trabajar de manera unida, promoviendo las relaciones entre los estudiantes (Piezon y Ferree, 2008; Hernández y Martín, 2017). De esta forma, se generan comunidades de aprendizaje e interdependencia positiva (Erkens y Bodemer, 2019; Kim y Ketenci, 2019).

Autores como Tejada, Garay y Romero (2017), corroboran la relación existente entre el rendimiento académico y el trabajo colaborativo en entornos virtuales de aprendizaje. Se afirma, por tanto, que el trabajo colaborativo favorece que los estudiantes lleven a cabo una planificación más responsable de su aprendizaje y un mayor compromiso e implicación, mejorando el desempeño de las tareas y el rendimiento académico (Villalustre y Del Moral, 2010). Asimismo, esta estrategia didáctica y metodología de trabajo puede utilizarse para llevar a cabo una gamificación en el aula (García-Lázaro, 2019), permitiendo que el alumnado desarrolle competencias y habilidades comunicativas, de liderazgo, reflexión, empatía y negociación, asumiendo responsabilidades 
que incrementen la capacidad de reflexión e impulsen el desarrollo de las competencias transversales (Lerís et al., 2017; Gallardo-López, 2018).

Sin embargo, existen factores como la falta de implicación y experiencia de los estudiantes (Muuro et al., 2014), la falta de comunicación y orientación por parte del tutor, la diferencia horaria y geográfica, las comunicaciones no verbales o el número de integrantes por grupo, que afectan al desarrollo y consecución de esta herramienta pedagógica (Roberts y McInnerney, 2007). En esta línea, Brindley, Blaschke y Walti (2009) o Chang y Kang (2016), reflejan experiencias negativas por parte del alumnado derivado de la carga excesiva de trabajo por parte de algunos integrantes grupales, calificaciones que no reflejan la participación e implicación real, las críticas poco constructivas (Thompson y $\mathrm{Ku}, 2010$ ) o el retraso en la retroalimentación a causa del tiempo y la distancia. En este sentido, la heterogeneidad de los miembros del equipo, en relación con las características individuales (motivaciones académicas, situación familiar y laboral, compatibilidad horaria, etc.), influyen directamente en la eficacia y creatividad de los trabajos. A su vez, la formación de grupos no demasiado extensos podría ser otra característica que ayudaría a evitar este tipo de experiencias. Bridges y Hallinger (1995) sugirieron que cada grupo de aprendizaje no debería exceder de los seis miembros. Por su parte, Smith y Dirkx (2007) aconsejan no superar los cuatro miembros.

Por otro lado, Chang y Kang (2016) afirman que los docentes han de ser conscientes de que no todos los alumnos llegan a tener el mismo nivel de implicación, compromiso y responsabilidad. Derivado de esto, los procesos de evaluación se tornan esenciales. Autoevaluaciones y heteroevaluaciones por pares son aspectos que suelen motivar a los estudiantes ya que les permiten obtener diferentes calificaciones y/o evaluaciones basadas en su trabajo real (Hermoza, 2015; Roberts y McInnerney, 2007). Además, el papel del tutor como mediador y guía (Gros et al., 2009) se convierte en un pilar imprescindible. Las funciones del profesorado en contextos en línea resulta indispensable para lograr el desarrollo real del estudiante (Chang y Kang, 2016; Roberts y McInnerney, 2007). Dentro de todo este proceso de tutorización, la retroalimentación del docente, así como su participación activa, resulta fundamental, ya que permite al alumnado construir buenas redes de conocimiento y un correcto clima grupal (González-Romá, 2011).

Dentro de este contexto, en este artículo se analizan las principales ventajas y desventajas, así como los procesos organizativos internos que, 99 estudiantes de los Grados de Educación Infantil y Educación Primaria de la Universidad 
Isabel I identificaron en la realización del trabajo colaborativo en entornos en línea. Dos preguntas de investigación guiaron este análisis:

1. ¿Cuáles son las principales ventajas y desventajas que los estudiantes identifican en los trabajos colaborativos en contextos en línea?

2. ¿Cómo se organizan internamente los estudiantes para el desarrollo de los trabajos colaborativos en contextos en línea?

\section{Metodología}

Los resultados de este artículo se enmarcan en un estudio desarrollado en la Universidad Isabel I, durante el curso académico 2016/2017. El objetivo principal del estudio era conocer, desde la perspectiva de los estudiantes que cursan los Grados de Magisterio de Infantil y Educación Primaria de forma en línea, las principales ventajas e inconvenientes que detectaban en la realización del trabajo colaborativo en entornos virtuales de Enseñanza Superior. De manera más específica, se propuso: 1. Analizar las ventajas y desventajas del trabajo colaborativo en línea; 2. Conocer los procesos organizativos internos de los estudiantes respecto al trabajo colaborativo en línea, y 3. Realizar propuestas de mejoras académicas hacia los docentes para el trabajo colaborativo en línea. Este artículo se ha centrado en analizar el primer y segundo objetivo del trabajo. Así, de forma más específica, se ha pretendido indagar sobre los procesos organizativos internos, el clima de trabajo, los procedimientos de comunicación establecidos, así como los protocolos de heteroevaluación desarrollados por los propios estudiantes implicados en el trabajo colaborativo.

El método de investigación utilizado en este estudio ha sido de carácter cualitativo. Se realizó un análisis de contenido de las entrevistas realizadas, con la intención de descubrir los componentes básicos del fenómeno estudiado, extrayendo la información relevante y conveniente, según los objetivos de investigación establecidos.

\subsection{Contextualización del estudio}

Los participantes de este estudio pertenecen a la titulación del Grado de Educación Infantil y Grado de Educación Primaria de la Universidad Isabel I. La población total de estudiantes de ambas titulaciones está formada por 815 alumnos, de los cuales 274 están matriculados en el Grado de Educación Infantil, 504 en el Grado de Educación Primaria y 37 en la doble titulación (Universidad Isabel I, 2017). 
Para la elección de la muestra se ha realizado una «selección basada en criterios» (Goetz y LeCompte, 1988). Para ello y, persiguiendo un muestreo selectivo, se ha atendido a unos rasgos, criterios y características relevantes que ha permitido identificarlos (Cotán, 2017). Concretamente, se han marcado seis criterios esenciales: titulación, modalidad de estudio en línea, estudiar en instituciones de Educación Superior, experiencia en la realización de trabajos colaborativos, disponibilidad para participar y voluntariedad.

La muestra final se compone de un total de 99 estudiantes, de los cuales 45 estudiaban el Grado de Educación Infantil, 51 el Grado de Educación Primaria y 3 el doble Grado de Educación Infantil y Primaria. Todos los participantes se encontraban en el primer y segundo curso de la titulación (ver detalles en tabla 1). Todos estos estudios fueron cursados en línea.

Tabla 1. Alumnado matriculado por curso y titulación

\begin{tabular}{lcc}
\hline & Primer curso & Segundo curso \\
\hline Grado de Educación Infantil & 27 & 18 \\
Grado de Educación Primaria & 29 & 22 \\
Grado de Educación Infantil y Primaria & 1 & 2 \\
\hline
\end{tabular}

Referente al género, 80 son mujeres y 18 hombres. Sus edades oscilaban entre los 22 y los 50 años, situándose un gran número de los participantes entre los 33 y los 41 ańos.

Las asignaturas cursadas en las que los 99 participantes se encontraban durante la recogida de información, debemos indicar que corresponden al segundo y tercer trimestre del curso 2016/2017. Concretamente, dichas asignaturas fueron: Didáctica de la Educación en Educación Infantil; Didáctica de la Educación en Educación Primaria; Psicología del Aprendizaje e Innovación y Proyectos Educativos.

Sobre la tipología de trabajos, cada asignatura ofreció una modalidad de trabajo: unidades didácticas, glosarios de términos, ensayos críticos y WebQuest. Todos ellos tuvieron una duración de cuatro semanas. Para finalizar, cabe indicar que los grupos de trabajo y su composición era diferente en cada asignatura, siendo cinco el número de estudiantes medio por cada grupo (ver tabla 2). Dichos grupos fueron elaborados por los docentes de manera aleatoria a través del campus virtual. En este mismo espacio, se informó al alumnado sobre sus integrantes y se abrió un foro compartido con el docente (por cada grupo) donde poder comunicar las dudas y/o consultas. 
Tabla 2. Tipologías y grupos de trabajo por asignaturas.

\begin{tabular}{llccc}
\hline \multicolumn{1}{c}{ Asignatura } & Tipología trabajo & $\begin{array}{c}\text { No Com- } \\
\text { ponentes } \\
\text { grupales }\end{array}$ & $\begin{array}{c}\text { No grupos } \\
\text { Educación } \\
\text { Infantil }\end{array}$ & $\begin{array}{c}\text { No grupos } \\
\text { Educación } \\
\text { Primaria }\end{array}$ \\
\hline Didáctica de la Educación & Unidad didáctica & 6 & 6 & 9 \\
\hline Psicología del Aprendizaje & Ensayo & 4 & 6 & 8 \\
\hline $\begin{array}{l}\text { Innovación y Proyectos } \\
\text { Educativos en Educación }\end{array}$ & $\begin{array}{l}\text { Glosario de términos } \\
\text { y WebQuest }\end{array}$ & 4 & 5 & 9 \\
\hline
\end{tabular}

\subsection{Validación del instrumento y recolección de datos}

Para la recogida de datos se utilizó, como instrumento de obtención de información, la entrevista estructurada. Dicha entrevista, fue diseñada y validada mediante juicio de expertos (Cabero y Llorente, 2013). Para ello, inicialmente se establecieron unos criterios de selección de expertos basados en: a) formación académica afín a la temática de estudio (pedagogos, psicopedagogos y docentes de Educación Superior); b) experiencia docente relacionada con el objeto de estudio, y c) experiencia investigadora sobre Educación Superior y entornos virtuales de aprendizaje.

A continuación, se diseñó una rúbrica de valoración del instrumento de investigación para que indicaran los aspectos estructurales de fiabilidad y validez del instrumento, así como las modificaciones de contenido que consideraran pertinentes. Concretamente, se quiso conocer la opinión del experto sobre la adecuación, claridad y extensión de los ítems de la entrevista, así como la pertinencia de las dimensiones analizadas.

Tras una primera revisión por parte de los expertos consultados, se indicaron leves modificaciones que se aplicaron antes de la distribución de la entrevista a la muestra seleccionada. En líneas generales, la entrevista fue valorada como apta, consiguiendo alcanzar los objetivos planteados a priori, siendo considerada como válida para la población estudiantil a la que iba dirigida.

El instrumento, compuesto por 14 ítems divididos en ocho dimensiones de estudio, fue administrado y cumplimentado de forma en línea, utilizando Google Forms. Concretamente, las categorías o dimensiones de análisis establecidas fueron: datos personales, modalidad del trabajo colaborativo, organización del trabajo y procesos de comunicación, heteroevaluación, implicación personal, clima de trabajo, beneficios y desventajas del trabajo colaborativo en línea, y propuestas de mejora (Tabla 2). 
Tabla 3. Estructura de la entrevista.

\begin{tabular}{|c|c|}
\hline Dimensión & Descripción de la dimensión \\
\hline 0. Datos personales & $\begin{array}{l}\text { Todos los datos personales recogidos durante los cuestionarios: } \\
\text { sexo, edad, titulación }\end{array}$ \\
\hline $\begin{array}{l}\text { 1. Modalidad Trabajo } \\
\text { Colaborativo }\end{array}$ & $\begin{array}{l}\text { Describir en qué se basaba el trabajo colaborativo. Temática, } \\
\text { modalidad y número de participantes }\end{array}$ \\
\hline 2. Organización & $\begin{array}{l}\text { Indicar cómo se ha organizado y planificado las tareas en el tra- } \\
\text { bajo, así como las vías y mecanismos de comunicación utilizados } \\
\text { para mantener el contacto y traspaso de contenido }\end{array}$ \\
\hline 3. Heteroevaluación & Importancia de la evaluación entre compañeros \\
\hline 4. Implicación personal & Formas de implicación y roles adquiridos en el grupo \\
\hline 5. Clima de trabajo & $\begin{array}{l}\text { Describir el clima de trabajo que se ha mantenido a lo largo del } \\
\text { desarrollo del trabajo }\end{array}$ \\
\hline $\begin{array}{l}\text { 6. Beneficios del TB } \\
\text { on-line }\end{array}$ & $\begin{array}{l}\text { Qué tipos de beneficios identifica el alumnado en la realización } \\
\text { del trabajo colaborativo en la modalidad on-line }\end{array}$ \\
\hline $\begin{array}{l}\text { 7. Desventajas del TB } \\
\text { on-line }\end{array}$ & $\begin{array}{l}\text { Qué tipos de desventajas identifica el alumnado en la realización } \\
\text { del trabajo colaborativo en la modalidad online, así como los prin- } \\
\text { cipales inconvenientes localizados durante el desarrollo del trabajo }\end{array}$ \\
\hline $\begin{array}{l}\text { 8. Propuestas de } \\
\text { mejoras }\end{array}$ & $\begin{array}{l}\text { Sugerencias para mejorar la organización y planificación del tra- } \\
\text { bajo colaborativo }\end{array}$ \\
\hline
\end{tabular}

Todas las entrevistas fueron de carácter anónimo y los únicos datos personales que se solicitaron a los estudiantes fueron: titulación, sexo y edad.

\subsection{Análisis de los datos}

El análisis de los datos se realizó desde una perspectiva estructural. Se debe indicar que el sistema de codificación utilizado para analizar la información recopilada fue el propuesto por Miles y Huberman (1994), teniendo en cuenta sus diferentes fases en el proceso de análisis de contenido: recopilación de los datos, establecimiento de categorías, interpretación y estructuración de los datos, y desarrollo de conclusiones (Cotán et al., 2020).

Para el análisis y codificación de los datos, se establecieron siete categorías: 1. Datos Personales; 2. Organización; 3. Roles desempeñados; 4. Heteroevaluación; 5. Implicación Personal; 6. Clima de trabajo; 7. Comunicación. A partir de todas las transcripciones de las entrevistas, se llevó a cabo el análisis del contenido asignando distintos códigos pertenecientes a las categorías previamente establecidas (Kuckartz y Rädiker, 2019). Para ello, se utilizó el programa informático de análisis de datos cualitativos MAXQDA (versión 2018). 


\section{Resultados}

A continuación, se presenta los resultados obtenidos en este estudio. Se han excluido del análisis los datos relativos a la edad o al sexo de las personas participantes al no considerarse relevantes para los resultados de la investigación. La muestra está formada en su gran mayoría por mujeres, y la media de edad es de 36 años. La información obtenida se ha dividido en tres bloques de análisis para facilitar la exposición de las principales temáticas abordadas: 1 . Organización y roles; 2. Heteroevaluación e implicación personal; 3. Clima de trabajo y vías de comunicación.

\subsection{Organización del trabajo colaborativo y roles desempeñados por los miembros del grupo}

Los estudiantes entrevistados manifestaron que el trabajo colaborativo fue realizado por grupos compuestos entre tres y seis participantes. El hecho de realizar el trabajo de manera colaborativa con otros estudiantes implicó que, en algunas ocasiones, surgiesen conflictos al no distribuirse las tareas de forma equitativa entre los miembros del grupo, y que la participación de unos y otros, fuese desigual.

«Dentro de los trabajos colaborativos que he hecho en lo que llevamos de curso, normalmente todos los miembros se han implicado, pero en uno de los de este trimestre he coincidido con compañeros que no se han implicado tanto e incluso que en algunos casos no han sido tolerantes con las propuestas de los demás» (Estudiante 5).

Por otro lado, consideraron que sería beneficioso que el grupo estuviese compuesto por un máximo de cuatro integrantes lo que facilitaría la comunicación en el trabajo. Respecto a la distribución de las tareas y actividades dentro del trabajo, de forma globalizada los estudiantes indicaron que se dividían los apartados realizándolos de manera individual o grupal y que, al término de los mismos, los unificaban y lo enviaban al espacio virtual. La mayoría de los grupos manifestaron que los repartos fueron equitativos.

«Hemos dividido los puntos de la Unidad Didáctica y cada uno se ha encargado de elaborar uno o dos, dependiendo de la extensión de cada uno. Las actividades normalmente las hemos diseñado entre todos» (Estudiante 8).

En algunos casos, la organización de las actividades, así como el reparto de tareas, se realizaba en función de las preferencias individuales o la experiencia y/o conocimiento del estudiante. 
«Las actividades se dividían en base a los conocimientos de cada uno de los miembros del grupo, y ayudándonos cuando alguno no sabía hacer algo» (Estudiante 27).

Un recurso planificativo que fue considerado como positivo en la organización y clarificación de las tareas, fue la realización de un esquema donde señalaban los participantes, las partes asignadas y un breve resumen. De esta forma, consideraron que no había lugar a confusión.

"Generalmente hemos hecho un esquema del trabajo que había que hacer y las hemos repartido en apartados. Cada participante se ha ido asignando tareas. Alguien también se encargaba de darle cohesión al trabajo, otra persona de darle formato y otra persona de subirlo a la red» (Estudiante 34).

Muchos de los estudiantes indicaron que era importante realizar una lectura final del trabajo previo a la entrega. Por ello, aquellos aspectos que se estimaban esenciales de la tarea, lo llevaban a cabo de forma grupal. Otros grupos, para asegurar este criterio, responsabilizaban a un miembro del equipo para que revisara todo el trabajo y certificara que todas las partes estuvieran coordinadas, cohesionadas y siguiendo el mismo formato.

En cuanto a la asignación de los roles asumidos dentro del grupo, todos los participantes expresaron de forma tácita que no habían establecido roles concretos entre ellos. Indicaron que cada uno realizaba las actividades o tareas encomendadas y que consideraban que todos tenían el mismo rol dentro del grupo.

«No se han asignado roles como tal, cada uno da lo que puede de sí mismo» (Estudiante 2).

«Más que asumir roles, todos hemos adquirido una responsabilidad en el desarrollo del trabajo» (Estudiante 55).

Sin embargo, pese a mostrar este tipo de afirmaciones, había estudiantes que, sin tener asignado un rol concreto, tenían que supervisar las tareas encomendadas a otros compañeros o revisaban todo el trabajo final y lo subían al aula virtual. Por ello, algunos estudiantes opinaron que siempre había alguien que destacaba en el grupo denominándolo el «líder». Esta misma función, en otros casos fue identificado como «jefe» o "coordinador».

«No ha habido asignación específica, puede que dos personas hayamos llevado más el peso, pero por ser más constantes en cuanto al tiempo de trabajo y las ganas por dejarlo hecho» (Estudiante 13). 
En otros casos, algunos participantes se identificaron como mediadores del grupo cuando surgía algún conflicto entre los propios compañeros. Sin embargo, todos ellos coincidieron en especificar que, dependiendo del trabajo, del grupo y de la asignatura, desempeñaron roles diferentes.

"No se han asignado roles, han ido surgiendo de forma espontánea. Dependiendo del grupo el rol ha sido uno u otro. A veces he llevado la voz cantante y otras veces no. No sé, me cuesta saber el rol que he ocupado en cada grupo, pero no siempre ha sido el mismo» (Estudiante 76).

En definitiva, en ningún caso los estudiantes identificaron ni asignaron de forma clara y explícita los roles grupales. Aunque, vieron reflejadas algunas funciones, especialmente la de dirección, organización o liderazgo. Asimismo, aseguraron que el uso de recursos y herramientas, como esquemas o mapas, les ayudaba a tener una mejor organización en el reparto de tareas.

\subsection{Importancia de los procesos de heteroevaluación e implicación personal}

En cuanto a los procesos de heteroevaluación, se pretendía conocer la implicación y esfuerzo que habían observado en sus compañeros de trabajo los estudiantes. Para ello, el docente estableció un porcentaje de la calificación (40\%) a la heteroevaluación realizada por los estudiantes. Dicho documento, consistía en una rúbrica, alojada en el campus virtual. Este instrumento, compuesto por ocho ítems, pretendía evaluar a través de un método mixto (cuantitativo y cualitativo), la calificación que el alumnado otorgaba a su compañero y el motivo. Para ello analizaba la implicación, participación, rendimiento y colaboración.

Para su realización, el alumnado descargaba dicho documento, lo cumplimentaba y lo volvía a subir al campus virtual. Este documento solo era entregado al docente y tenía carácter anónimo dentro del grupo. Al finalizar el trabajo, de manera individual, al alumnado se le ofrecía información sobre la calificación media obtenida (tanto de la heteroevaluación como en la evaluación del propio docente) y retroalimentación general del porqué.

En consonancia, referente a este instrumento, se encontraron respuestas diversas. Algunos estudiantes que mostraban su disconformidad con este tipo de evaluación por varios motivos, como la calificación final obtenida, la falta de revisión del trabajo o la fiabilidad y validez del propio sistema de evaluación.

«Considero que es bueno realizar trabajos colaborativos, pero después de mi última experiencia, se me quitan las ganas de participar, porque mi esfuerzo no ha servido para nada» (Estudiante 22). 
Asimismo, algunos participantes consideraban que este tipo de acción evaluativa no era relevante, y que podía perjudicar de forma negativa al clima grupal. Sin embargo, la mayoría de los participantes sí estaba de acuerdo con la heteroevaluación, ya que manifestaron que no todos los participantes se involucraban al mismo nivel y, por ello, consideraban adecuado que entre ellos pudieran analizarse y evaluarse.

«Creo que es importante porque no todo el mundo se implica igual y no es justo que todos tengamos la misma nota» (Estudiante 5).

En este sentido, opinaban que gracias a este tipo de evaluación podían obtener una calificación coherente con el nivel de participación de cada miembro del grupo, así como aumentar su nivel de esfuerzo e implicación. Consideraban que este tipo de acción evaluativa invitaba a la participación activa, aumentaba el nivel de responsabilidad individual y grupal, y permitía conocer la participación real de cada miembro del grupo, así como mejorar y perfeccionar las técnicas y habilidades de trabajo en equipo.

«Sí, porque reconoce el esfuerzo de cada uno e invita a la participación activa» (Estudiante 63).

Según las manifestaciones de los estudiantes, se puede afirmar que este tipo de evaluación ayudaba a los participantes a conocer aspectos de mejora y críticas constructivas.

«En el caso de los trabajos colaborativos me parece imprescindible que unos compańeros evalúen a los demás miembros del grupo de forma individual, pues no todos se implican ni participan, ni respetan las normas que se puedan llegar a establecer» (Estudiante 86).

Sin embargo, el desconocimiento de algunos ítems o la subjetividad de los mismos no permitía realizar una evaluación real y objetiva de la labor y de la actividad desarrollada de forma individual. Entre las opciones de mejora para la evaluación, los participantes aportaron la heteroevaluación anónima como opción que les permitiría expresar su opinión y evitar calificaciones injustas y sobrevaloradas.

«Creo que es importante, pero debería poder hacerse de forma anónima porque de lo contrario, todos los compañeros acaban puntuando el máximo para conseguir una mejor calificación cuando hay ocasiones en que algunos compańeros deberían ser evaluados peor por su escasa colaboración y participación en los trabajos o la escasa calidad de sus aportaciones» (Estudiante 47). 
No obstante, algunos estudiantes indicaron que, con independencia de la evaluación entre compañeros, si el grupo tenía algún problema se lo transmitían al docente para que pudieran actuar en consonancia con las informaciones del mismo.

Finalmente, todos los participantes coincidieron en afirmar que la evaluación entre pares es un aspecto importante dentro del desarrollo del trabajo colaborativo ya que les permitía no solo evaluar el grado de desarrollo y trabajo de cada miembro sino también aprender, reflexionar y mejorar sobre su propia praxis educativa.

«Creo que todos hemos de evaluarnos para mejorar y aprender» (Estudiante 9).

Otro de los aspectos relacionados con la evaluación positiva del alumnado radica en el nivel de implicación y responsabilidad en el desarrollo del trabajo colaborativo.

«Sí, porque al ser un trabajo grupal en el que la nota final del mismo es para todos la misma, es necesaria una implicación igualitaria de todos los miembros del mismo, y en su defecto, reducir esa calificación» (Estudiante 42).

De manera general, los estudiantes respondieron que estos aspectos eran la clave para el éxito de la tarea. Sin embargo, los motivos de dicha afirmación variaban en función de los intereses y motivaciones personales. Mientras algunos estudiantes aseguraron que es importante su implicación grupal en el trabajo, debido al aprendizaje que obtienen de él y la relación práctica que pueden establecer con la teoría, otros estudiantes respondieron que participaron activamente para poder obtener calificaciones positivas.

«Me he implicado en el trabajo porque creo que es importante para el logro de los objetivos y competencias establecidas. Es una forma de evidenciar la teoría en ejemplos prácticos» (Estudiante 44).

Además, consideraron que este tipo de actividades eran esenciales en la formación del Grado de Educación. Indicaron que cuando se incorporasen al mundo laboral, como futuros docentes tendrían que saber trabajar en equipo con otros compañeros, familias y/o asociaciones. Por ello, esta tarea les permitió adquirir habilidades de comunicación y de trabajo en equipo.

«Sí. El profesorado debe ser capaz de trabajar en equipo. Serán situaciones reales en los centros, con otros maestros/padres, dirección/asociaciones... Tenemos que saber transmitir la comunicación, dialogar y trabajar en equipo» (Estudiante 88). 
En este aspecto, algunos estudiantes mostraron la importancia de implicarse en los trabajos colaborativos dando prioridad al objetivo común frente al individual. Además, indicaron la relevancia de establecer las fechas y plazos de entrega de forma grupal y consensuada. Por ello, algunos estudiantes expresaron su compromiso con el trabajo ya que ellos mismos habían indicado su derecho tácito a participar, así como su interés hacia la temática y el respeto hacia sus compañeros.

«Sí, primero porque es un tema que me gusta y segundo porque se lo debo al grupo. Cuando uno decide realizar un trabajo colaborativo su deber es implicarse y colaborar en todo lo posible» (Estudiante 27).

Por otro lado, la implicación real de los estudiantes no solía ser la misma en todos los trabajos ya que las circunstancias personales, laborales y familiares incidían en este aspecto. Por ello, los estudiantes consideraron imprescindible ser constante en todas las partes del trabajo, siendo primordial que el trabajo tuviese una cohesión y coordinación en todas sus partes.

\subsection{Clima de trabajo y vías de comunicación}

Las opiniones con relación al clima de trabajo fueron variadas. Por un lado, y con carácter generalizado, se pueden encontrar aquellos grupos cuyo ambiente ha sido positivo puesto que las motivaciones y objetivos eran similares entre los miembros. El hecho de coincidir en metas similares, hizo que las relaciones del grupo fueran cordiales.

«Normalmente suele haber muy buen ambiente ya que todos deseamos hacer un gran trabajo y conseguir una buena nota» (Estudiante 78).

Aspectos como la implicación, responsabilidad, autocrítica, empatía, apoyo y ayuda mutua fueron valorados positivamente por los estudiantes e incidieron de forma satisfactoria en las relaciones grupales.

«Muy bueno, de apoyo mutuo, se ha mostrado iniciativa, aceptación de los errores propios, apertura hacia las ideas de los demás... enriquecedor» (Estudiante 34).

Sin embargo, también hubo participantes que aseguraron que las relaciones no fueron positivas, bien con todos los miembros del grupo o con un porcentaje significativo del mismo. Condicionantes como el estrés, la realización de varias tareas al mismo tiempo o los exámenes fueron aspectos que repercutieron de manera negativa en el clima grupal. 
«Hay clima de compañerismo, pero al mismo tiempo de estrés por la falta de tiempo para la realización del mismo justo antes de los exámenes» (Estudiante 93).

Asimismo, las decisiones individuales que algunos de los estudiantes tomaron respecto al trabajo, como la poca implicación en las actividades o las críticas poco constructivas entre compañeros, también fueron aspectos negativos que incidieron en el clima de las relaciones grupales.

«Fue por momentos un poco tenso e incómodo. La falta de comunicación, la poca implicación y la poca capacidad de autocrítica hace que el clima no sea agradable» (Estudiante 26).

Por todo ello, se puede afirmar que la implicación, la responsabilidad, la realización de las tareas, el respeto hacia la programación, la empatía o la colaboración entre todos los miembros del grupo de trabajo son indicadores de un buen clima grupal.

Uno de los factores destacados para un buen clima fue la comunicación. Las vías de comunicación utilizadas para la transmisión de información y contenido fueron diversas: foro del aula virtual, Hangouts, WhatsApp, E-mail o Google Drive. Sin embargo, la aplicación WhatsApp fue la más usada por todos ellos ya que indicaron que era la más accesible, rápida y fácil de usar.

«Por la plataforma de foro habilitada por los profesores, por e-mail, aunque lo más cómodo es WhatsApp por la velocidad de emisión/recepción de los mensajes» (Estudiante 45).

Con relación al uso del foro de la asignatura, varios de los participantes mostraron su disconformidad con el mismo ya que indicaban que ralentizaba los procesos de comunicación.

"WhatsApp, aunque se pedía que fuese por el foro. Todos los miembros del grupo pensamos que el foro es bastante incómodo para poder tener una comunicación efectiva» (Estudiante 10).

Sin embargo, aseguraron que la mayoría de ellos dejaban constancia de sus decisiones en el foro de la asignatura para que el docente estuviera al tanto de las mismas, así como de la implicación en el trabajo de los participantes del grupo.

«Se pedía que fuese por el foro. Todos los miembros del grupo pensamos que el foro es bastante incómodo para poder tener una comunicación efectiva» (Estudiante 9). 
Igualmente, aseguraron que el principal medio o mecanismo para enviar los avances del trabajo, así como las partes que conforman el mismo, fue Google Drive, ya que era una herramienta donde todos podían ver sus avances y actualizar los archivos. Esta opción, en algunos casos fue sustituida por Dropbox y, en otras, se vio complementada con el uso del correo electrónico.

«Hemos trabajado con Google Drive, viendo las aportaciones y avances de cada participación» (Estudiante 55).

No obstante, en contadas ocasiones algunos participantes revelaron que también usaron la vía del WhatsApp para indicar los resultados del trabajo a los compañeros que no disponían de acceso al aula. Tan solo un grupo indicó que realizó en su totalidad el trabajo colaborativo en el espacio creado y habilitado para tal fin en el aula virtual.

\section{DisCUSIÓN Y CONCLUSIONES}

El espacio de Educación Superior modifica los roles tradicionales, centrando el proceso de aprendizaje en el estudiante y reconociendo tanto su trabajo presencial como no presencial. Este modelo abre enfoques alternativos, como los basados en aprendizajes colaborativos, que fomentan la reflexión, la autonomía y el dinamismo en las prácticas docentes (Cotán et al., 2020; Yadegaridehkordi et al., 2019; Zhou et al., 2019). El papel del estudiante también cambia convirtiéndose en protagonista y responsable tanto de su aprendizaje como el de sus compańeros, de manera colaborativa (Erkens y Bodemer, 2019; Kim y Ketenci, 2019).

Dentro de este contexto, una de las conclusiones derivadas del artículo fue la referida al número de participantes en los grupos. Bajo este criterio, los grupos formados por cuatro personas permitían una distribución equitativa de tareas, así como tener mayor espacio para la participación (Smith y Dirkx, 2007). Así, generar grupos con inquietudes y motivaciones similares, mejoraría de forma directa la eficacia, creatividad y clima grupal de los trabajos (Bridges y Hallinger, 1995; Piezon y Ferree, 2008; Roberts y McInnerney, 2007). La diversidad y distribución de roles y liderazgos, se consideró esencial para la gestión y el desarrollo correcto del trabajo colaborativo (Brindley et al., 2009; Chang y Kang, 2016; Lerís et al., 2017).

En segundo lugar, la realización de una heteroevaluación anónima fue considerada una herramienta de reflexión, crítica y mejora. Este tipo de evaluación permitió a los estudiantes valorar el trabajo real de cada componente (Roberts y McInnerney, 2007). Igualmente, esta acción propició la participación activa, aumentando, por ende, el nivel de responsabilidad individual y grupal. 
Con este tipo de acciones evaluativas, los estudiantes incrementaron la comprensión del significado de la evaluación y las repercusiones de su propio rendimiento. Sin embargo, los datos mostrados reflejan la importancia de formar a los estudiantes en procesos de heteroevaluación (Hermoza, 2015). Este hecho permitiría al estudiante poder evaluar a sus compañeros partiendo de unos conocimientos mínimos previos. Relacionado con ello, una de las propuestas de mejora que emergió durante la recogida de datos, fue la creación de un contrato de implicación y responsabilidad grupal (Cotán et al., 2020). Los estudiantes consideraron que esta acción mejoraría notablemente el grado de implicación y de responsabilidad de los integrantes (Villalustre y Del Moral, 2010). De esta forma, se fomentaría un desarrollo óptimo y enriquecedor del proceso de enseñanza-aprendizaje.

En este orden de ideas, la implicación y la responsabilidad grupal fueron aspectos de lo más relevantes para los estudiantes. Estas cuestiones se convirtieron en transcendentales para una correcta organización y desarrollo de este (Brindley et al., 2009; Muuro et al., 2014). Sin embargo, en este estudio se manifiesta como todos los participantes no tienen las mismas metas y objetivos, generando una clara desigualdad en la realización de las tareas (Chang y Kang, 2016). Conclusiones similares se obtuvieron en los trabajos de Roberts y McInnerney (2007) o Piezon y Ferree (2008).

En este sentido, se ha identificado el clima grupal como un factor importante en la evolución de la actividad (González-Romá, 2011; Thompson y Ku, 2010). Aspectos como la motivación, la responsabilidad, autocrítica, empatía, apoyo y ayuda mutua fueron características positivas que generaban un buen clima de trabajo grupal (Gros et al., 2009). Además, tal y como se ha visto reflejado en este estudio, el clima de trabajo incide de forma directa en los resultados de este (González-Romá, 2011; Peñazola, 2017).

Otra de las conclusiones obtenidas es la buena comunicación como sinónimo de clima positivo. Comunicaciones asíncronas y síncronas con carácter asiduo y continuo fueron consideradas fundamentales para el correcto desarrollo del trabajo. Es indudable que las comunicaciones generadas a través de los grupos de WhatsApp actúan como verdaderas redes sociales donde se intercambia información (Barrio y Ruiz, 2017). Por ello, los docentes universitarios podrían aceptar esta herramienta que se instaura en el aula y que sirve para establecer vínculos interrelacionales y, por ende, transmitir contenidos. Así, el uso del WhatsApp resulta motivador para los estudiantes, siendo una tecnología que les permite organizarse de forma muy práctica. Igualmente, el uso de otras plataformas como Google Drive o Dropbox fueron los medios principales para la realización y puesta en común de los avances del trabajo colaborativo. 
Dentro de este contexto, se afirma que el trabajo colaborativo en entornos en línea en Educación Superior es destacado por los estudiantes como una actividad y una herramienta pedagógica y metodológica importante. Esta estrategia permite que, el alumnado, adquiera nuevas visiones y perspectivas (Roberts y McInnerney, 2007) así como poner en práctica habilidades sociales $\mathrm{y}$ contenidos teóricos que son fundamentales para su futuro profesional como docentes.

\section{REFERENCIAS BIBLIOGRÁFICAS}

Algaba, E. (2015). Universidad pública y privada en España: dos modelos distintos con objetivos similares. Encuentros Multidisciplinares, 49, 1-10.

Barrio, Á. y Ruiz, I. (2017). Hábitos de uso del Whatsapp por parte de los adolescentes. INFAD Revista de Psicología, 1, 23-30. https://doi.org/10.17060/ ijodaep.2017.n1.v2.915

Bridges, E. y Hallinger, P. (1995). Implementing Problem Based Learning in Leadership Development, ERIC Clearinghouse on Educational Management, University of Oregon, Eugene.

Brindley, J., Blaschke, L.M. y Walti, C. (2009). Creating effective collaborative learning groups in an online environment. The International Review of Research in Open and Distributed Learning, 10(3), 1-18. https://doi. org/10.19173/irrodl.v10i3.675

Cabero, J. y Llorente, Ma . C. (2013). La aplicación del juicio de experto como técnica de evaluación de las tecnologías de la información (TIC). Eduweb. Revista de Tecnología de Información y Comunicación en Educación, 7(2), 11-22.

Castellanos, J. C. y Niño, S. A. (2020). Aprendizaje colaborativo en línea, una aproximación empírica al discurso socioemocional de los estudiantes. Revista Electrónica de Investigación Educativa, 22, e20, 1-12. https://doi. org/10.24320/redie.2020.22.e20.2329

Chang, B. y Kang, H. (2016). Challenges facing group work online. Distance Education, 37(1), 73-88. https://doi.org/10.1080/01587919.2016.1154781

Cotán, A. (2017). Educación inclusiva en las instituciones de educación superior: narrativas de estudiantes con discapacidad. Revista Española de Discapacidad, 5(1), 43-61. https://doi.org/10.5569/2340-5104.05.01.03

Cotán, A., Martínez, V., García-Lázaro, I., Gil-Mediavilla, M. y Gallardo-López, J.A. (2020). El trabajo colaborativo online como herramienta didáctica en Espacios de Enseñanza Superior (EEES). Percepciones de los estudiantes de los Grados en Educación Infantil y Primaria. Revista d'Innovació Docent Universitària, 12, 82-94. https://doi.org/10.1344/RIDU2020.12.9 
Erkens, M. y Bodemer, D. (2019). Improving collaborative learning: Guiding knowledge exchange through the provision of information about learning partners and learning contents, Computers and Education, 128, 452-472. https://doi.org/10.1016/j.compedu.2018.10.009

Gallardo-López, J. A. (2018). Educating in emotional competences in the information and communication society. In M. Reyes-Tejedor, J. GómezGalán, J.L. Sarasola y E. Meneses. Values Education in the Digital Age: An Action Training and Research Approach. UMET.

Gallardo-López, J. A. y López-Noguero, F. (2020). Twitter como recurso metodológico en Educación Superior: Una experiencia educativa con estudiantes de Trabajo Social. Alteridad, 15(2), 174-189. https://doi.org/10.17163/ alt.v15n2.2020.03

García-Lázaro, I. (2019). Escape room como propuesta de gamificación en educación. Revista Educativa Hekademos, 27, 71-79. Recuperado de https:// www.hekademos.com/index.php/hekademos/article/view/17

Goetz, J. P. y LeCompte, M. D. (1988). Etnografía y diseño cualitativo en investigación educativa. Madrid, Ediciones Morata.

González, J. y Wagenaar, R. (2003). TUNING. Educational Structures in Europe. Recuperado de http://tuningacademy.org/wp-content/uploads/2014/02/ TuningEUI_Final-Report_SP.pdf

González-Romá, V. (2011). El clima de los equipos de trabajo: una propiedad configuracional, Papeles del Psicólogo, 32(1), 48-58.

Gros, B., García, I. y Lara, P. (2009). El desarrollo de herramientas de apoyo para el trabajo colaborativo en entornos virtuales de aprendizaje. RIED, 12(2), 115-138. https://doi.org/10.5944/ried.2.12.903

Hermoza, L. M. (2015). La evaluación formativa-colaborativa para la construcción del aprendizaje en los alumnos de arte y diseño. Educación, 24(46), 47-72.

Hernández, A. y Martín, J. (2017). Concepciones de los docentes no universitarios sobre el aprendizaje colaborativo con TIC. Educación XX1, 20(1), 185-208. https://doi.org/10.5944/educxx1.17508

Hernández, J. y Pérez, J.A. (2019). La Universidad Española en Cifras. CRUE Universidades Españolas.

Kim, M.K. y Ketenci, T. (2019). Learner participation profiles in an asynchronous online collaboration context. Internet and Higher Education, 41, 62-76. https://doi.org/10.1016/j.iheduc.2019.02.002

Korhonen, A. M., Ruhalahti, S. y Veermans, M. (2019). The online learning process and scaffolding in student teachers' personal learning environments. Education and Information Technologies, 24(1), 755-779. https://doi.org/ 10.1007/s10639-018-9793-4 
Kuckartz, U. y Rädiker, S. (2019). Analyzing Qualitative Data with MAXQDA. Text, Audio and Video. Switzerland, AG: Springer. https://doi.org/10.1007/ 978-3-030-15671-8

Lerís, D., Letosa, J., Usón, A., Allueva, P. y Bueno, C. (2017). Trabajo en equipo y estilos de aprendizaje en la educación superior. Revista Complutense de Educación, 28(4), 1267-1284. https://doi.org/10.5209/RCED.51722

Marín, V. I., Asensio-Pérez, J. I., Villagrá-Sobrino, S., Hernández-Leo, D. y García-Sastre, S. (2018). Supporting online collaborative design for teacher professional development. Technology, Pedagogy and Education, 27(5), 571-587. https://doi.org/10.1080/1475939X.2018.1547787

Miles, M. B. y Huberman, A. M. (1994). Qualitative data analysis. Beverly Hills, CA: Sage Publications.

Molina, P. (2013). Collaborative education and autonomy concept in ICT for instructors in Higher Education. Academic journal and virtuality, 6(1), 53-61.

Mostrom, A. M. y Blumberg, P. (2012). Does learning-centered teaching promote grade improvement? Innovative Higher Education, 37(5), 397-405. https:// doi.org/10.1007/s10755-012-9216-1

Muuro, M. E., Wagacha, W. P., Kihoro, J. y Oboko, R. (2014). Students' perceived challenges in an online collaborative learning environment: A case of higher learning institutions in Nairobi, Kenya. The International Review of Research in Open and Distributed Learning, 15(6). https://doi.org/10.19173/irrodl. v15i6.1768

Peñazola, J. (2017). Incidencia del aprendizaje colaborativo en la práctica educativa. Revista de Didácticas Especificas, 16, 46-60.

Piezon, S. L y Ferree, W. D (2008). Perceptions of social loafing in online learning groups: A study of public university and U.S. naval war college students. International Review of Research in Open and Distributed Learning, 19(2). https://doi.org/10.19173/irrodl.v9i2.484

Roberts, T. S y McInnerney, J. M (2007). Seven problems of online group learning (and their solutions). Journal of Educational Technology y Society, 10(4), 257-268.

Santizo, C. (2016). Condiciones institucionales del trabajo colaborativo como estrategia de cambio del sistema educativo. Perfiles Educativos, 38(153), 154-167. https://doi.org/10.22201/iisue.24486167e.2016.153.57642

Smith, R. O. y Dirkx, J. M. (2007). Using consensus groups in online learning. New Directions for Adult and Continuing Education, 113, 25-35. https:// doi.org/10.1002/ace.244

Tejada, E., Garay, U. y Romero, A. (2017). La incidencia de la participación cooperativa en entornos virtuales en el rendimiento académico, Edutec: Revista 
electrónica de tecnología educativa, 60, 1-11. https://doi.org/10.21556/ edutec.2017.60.889

Thompson, L. y Ku, H. (2010). Degree of online collaboration and team performance: A case study, Quarterly Review of Distance Education, 11(2), 127-135.

Universidad Isabel I (2017), Informe Anual de Calidad de curso académico 2016/2017. Recuperado de https://www.uil.es/sites/default/files/page/ files/2017_informe_anual_calidad.pdf

Villalustre, L. y Del Moral, Ma . E. (2010). Innovaciones didáctico-metodológicas en el contexto virtual de Ruralnet y satisfacción de los estudiantes universitarios. Revista Iberoamericana sobre Calidad, Eficacia y Cambio en Educación, 8(5), 70-81.

Yadegaridehkordi, E., Shuib, L., Nilashi, M. y Asadi, S. (2019). Decision to adopt online collaborative learning tools in higher education: A case of top Malaysian universities. Education and Information Technologies, 24(1), 79-102. https://doi.org/10.1007/s10639-018-9761-z

Zhou, X., Chen, L. y Chen, C. (2019). Collaborative learning by teaching: A pedagogy between learner-centered and learner-driven, Sustainability, 11(4). https://doi.org/10.3390/su11041174 